\title{
Daily Living Tasks Affected by Sensory and Motor Problems in Children with Autism Aged 5-12 Years
}

\author{
Sheza Ahmed ${ }^{1}$, Humaira Waseem ${ }^{2}$, Ambreen Sadaf $^{3}$, Rabia Ashiq $^{4}$, Hafsa Basit $^{5}$, Sybil Rose $^{6}$ \\ Sheza.ahmed6@gmail.com ${ }^{1}$ \\ seehumairawaseem@gmail.com² \\ ambreensadafot@gmail.com ${ }^{3}$ \\ rabiaashiq36@gmail.com ${ }^{4}$ \\ hafsa.basit786@gmail.com ${ }^{5}$ \\ sybilgill36@gmail.com6
}

\begin{abstract}
Background:Children with Autism Spectrum Disorder faces sensory and motor problems (Vestibular, proprioceptive, Tactile, Auditory and visual processing). Due to these problems, individuals faces difficulties in performing their daily task properly.

Objective(s): The objective of this study was to determine how daily living (DL) tasks affected in children with autism Spectrum disorder having sensory and motor problems aged 5-12 years.

Methodology:The study conducted in Rehab care school for special children and Hamza Montessori School for special children. Data was collected by using purposing sampling technique in the duration of 4 months. Cross sectional study design was used. 60 patients from both genders were included. Sample selection criteria included the children with autism spectrum disorder with age range of 05-12 years. Sensory profile questionnaire based on Ayers sensory integration theory and Standardized Barthel index scale were used and the data was analyzed by SPSS to determine how daily living task are affected by sensory and motor problems.

Results:The results showed that Tactile and auditory processing disorders were highly affecting the performance of the children. About $70 \%$ of these individuals were having problems in performing their daily living tasks.

Conclusion(s): It was concluded that most of the children with autism spectrum disorder have sensory and motor problems. The performance in activities of daily living is affected by sensory and motor problems especially the tactile and auditory processing. Tactile processing shows that children have difficulty in performing activities like dressing, eating and toileting.
\end{abstract}

Keywords: ASD, DL, Sensory and motor problems

DOI: $10.7176 / \mathrm{JHMN} / 92-02$

Publication date:August $31^{\text {st }} 2021$

\section{INTRODUCTION}

Autism Spectrum Disorder is a persistent deficits in social communication and social interaction across multiple contexts, as manifested by the Deficits in social-emotional reciprocity, deficits in nonverbal communicative behaviors used for social interaction, abnormalities in eye contact and body language or deficits in understanding and use of gestures; to a total lack of facial expressions and nonverbal communication and deficits in developing, maintaining, and understanding relationships. ${ }^{1}$ These debilitations may make troubles for an individual perform task independently ${ }^{2}$. A developing societal concern, the commonness of Autism Spectrum Disorder (ASD) has about multiplied in the previous ten years. In 2008, the predominance of ASD was evaluated to be one in each 125 kids; nonetheless, as of the 2018 Autism and Developmental Disabilities Monitoring Report, the quantity of children determined to have ASD has expanded to one in each $59^{3}$. Late investigates evaluate that $96 \%$ of the youngsters with autism spectrum disorder have sensory difficulties ${ }^{4}$. The reason of autism spectrum disorder is as yet obscure. However, the analysts propose that genetics and ecological variables can assume significant job. ASD is neurodevelopmental issue that is very heritable ${ }^{5,6}$. A few investigations have recommended that ASD might be brought about by an epigenetic instrument (heritable change in the quality articulation without change in DNA grouping). Now it is increasingly evident that there is anything but a solitary reason for ASD. Its etiology is multifactorial ${ }^{7}$. The proof assessed has shown that sensory-motor issues are pervasive in autism spectrum condition and they have huge effect on a person's capacity performance ${ }^{8}$.Children Autism Spectrum Disorder having sensory motor problems show such hypo or hyper responses such as sensory interests, repetitions, and seeking behaviors HYPO is considered a lack of or delayed response to sensory stimuli (e.g. a lack of orienting to loud sounds, slow to react to pain) HYPER is defined by an exaggerated or avoidant response to sensory stimuli (e.g. distress during grooming, aversion to sounds) ${ }^{9}$ Studies have demonstrated that autism spectrum condition has a relationship with sensory reactivity and motor coordination and recommending that the advancement of correspondence and social abilities influenced by these sensory issues ${ }^{10}$. Sensorimotor problems includes the way receiving sensory messages (sensory info) and delivering a reaction (movement output). We get sensory information from our bodies and nature through our sensory system (vision, hearing, 
smell, taste, contact, vestibular, and proprioception) ${ }^{11}$. Sensory motor system gives a fundamental establishment to perception and activity just as numerous significant cognitive and social procedures all through life ${ }^{12}$. Many sensory-motor skills are set up during childbirth however keeps on quickly develop and rearrange all through the early child years ${ }^{13}$. Sufficient sensory regulation is expected to keep up fitting physiological excitement for selfsupport (for example eating, resting) to encourage advancement of self-controlling behaviors (for example Sucking to mollify, self-talk amid testing task) and to help persuasive system to participate in meaningful tasks 14. Poor sensor-motor integration has for some time been incriminate as a reason for motor issues in developmental disorder, for example, developmental coordination issue (DCD), ongoing examination has likewise researched sensory-motor deficiencies in children with attention deficit hyper activity disorder (ADHD) and autism spectrum disorder. Poor sensory-motor coordination has all the earmarks of being connected to DCD, yet not ADHD. Additionally, sensory-motor deficits in children with DCD and social problems may give understanding into a portion of the social challenges found in these groups of children ${ }^{15}$. Individuals with autism spectrum disorder regularly show an example of hyper or hypo-sensitivity to a sensory stimulus ${ }^{16}$. Numerous investigations have done as so far on behavioral area of autism spectrum disorder which centers on weaknesses in socialization, communication and comprehension. Anyway sensorimotor improvement and self-care stay little investigated when contrasted with different areas of development. Kids with autism spectrum disorder experience issues in freely performing self-care aptitudes which we call daily living skills (DLS) like dressing, washing, self-grooming and toileting independently ${ }^{17}$. The activities which we play out each day, the tasks of daily living are of two kinds; Basic activities of daily living and instrumental activities of daily living (IADL) 18. Children with ASD who have difficulties in performing fine motor activities, buttoning a sweater will turn into a test for them; they likewise don't care for the way some woolen stuff of clothing feel ${ }^{19}$. There are not many studies that research the effects of sensorimotor challenges on daily living skills in youngsters with autism spectrum disorder. It is critical to archive the connection among sensorimotor and everyday living aptitudes to all the more likely comprehend the particular needs of a child with autism spectrum disorder and to make feasible for them to coordinate in the social environment ${ }^{20}$. Tsameret Ricon, et al., (2017) conducted a study on relationship between sensory processing in children with high functioning autism spectrum disorder (HFASD) and their daily schedules which involves 20 kids aged 5-7 with high functioning autism spectrum disorder (HFASD) and 30 ordinarily developing age matched children having the equivalent financial foundation. A short sensory profile finished by the guardians from the two groups to examine their kids' sensory processing and sensory processing disorder. Data about youngsters' support and execution in tasks of daily living was acquired by Make My Day (MMD) list. Kids with advanced high functioning autism spectrum disorder (HFASD) were having altogether progressively tangible troubles because of which they face confinements in performing tasks of daily routine ${ }^{21}$. (Jeanne Zobel-Lachiusa et al., 2015) the study examined the meal time behaviours in children with autism spectrum disorder (ASD) and sensory differences. 34 kids with ASD and 34 normally developing children of same age were involved in this study. A youngster and parent report Performa was used and the outcome shows that the kids with autism spectrum disorder scored essentially extraordinary on the proportion of sensory differences and eating practices from the normally developing children. The examination helps the parental figure and advisor to recognize the issues of eating conduct related with sensory differences ${ }^{22}$. (Scott D. Tomchek., et al., 2015) the examination researches to what extent, particular sensory processing pattern identifies with the parts development for example social behavior, fine and gross motor skills, and commitment in activities of daily living and so forth. Members included 400 pre-school aged youngsters with autism spectrum disorder and the clinical information was gathered by a review outline survey. Results recommend that sensory processing patterns influence child's improvement and versatile behavior and motor skills ${ }^{23}$.

The purpose of this study is to assess the relationship between sensory motor development and activities of daily living. Independence in activities of daily living for a child with autism spectrum disorder is the main concern of the therapist and parents and it is important for child's participation in school and day care

\section{Methodology}

Research was conducted by using a questionnaire for the assessment of sensorimotor issues and Barthel index scale for the assessment of activities of daily. It is an observational study cross sectional study, conducted in private Rehab Care and Hamza Montessori for special children in Lahore. The duration of study was 4 months. 60 children diagnosed with Autism Spectrum Disorder (ASD) were included in the study whose age range was 5-12. The variables of the study were sensory motor issues that are vestibular system, proprioceptive problems, tactile problems, visual processing and auditory processing. The questionnaire was given to the parents and the results were scored. The range of scoring was 0-20. Then the total scores were compared for sensorimotor issues and daily living tasks. All the categorical variables were presented in the form of frequency and percentages and continuous variable in mean standard deviation SD or histogram. The relationship between sensorimotor problems and activities of daily living in children with autism spectrum disorder ASD were tested through Pearson co-relation. Data was analyzed with the help of SPSS 21version. 


\section{RESULTS}

The result selection is consisted of descriptive and inferential statistics. Percentage, frequencies, means and standard deviations were calculated for the demographics. Pearson correlation was used to assess correlation between sensory-motor issues and activities of daily living in children with autism spectrum disorder.

\section{Descriptive statistics}

Descriptive statistics were used to describe the demographical. Percentages and frequencies were taken for all demographic variables. Mean and standard deviations were calculated for the age of the participants, which was the only continuous variable.

The majority of the individual (28) were in the age range of 5-7 years, 23 individuals in the age range of 812 years and 9 individuals in the age range of 13-15 years and the majority of the individual (28) were male than female.

\begin{tabular}{|l|l|l|l|}
\hline Table 1: Frequency of sensory-motor problem in children with ASD & \multicolumn{2}{|l|}{ Yes } & No \\
\hline Variables & Sub variables & & 53 \\
\hline \multirow{4}{*}{ Vestibular problems } & & 7 & 47 \\
& Dislikes play & 13 & 7 \\
\cline { 2 - 4 } & Clumsy, stumbles & 53 & 22 \\
\cline { 2 - 4 } & Jumps on swing & 38 & 36 \\
\cline { 2 - 4 } & Spin constantly & 24 & 51 \\
\hline Proprioceptive problems & Stiff movements & 9 & 19 \\
\cline { 2 - 4 } & Difficulty in and out chair & 41 & 32 \\
\cline { 2 - 4 } & Pencil grasp & 41 & 32 \\
\hline Tactile problems & Dislikes textures & 34 & 36 \\
\cline { 2 - 4 } & Dislikes brushing, combing & 24 & 30 \\
\cline { 2 - 4 } & Craves touch & 30 & 19 \\
\cline { 2 - 4 } & React to painful stimuli & 41 & 25 \\
\hline Visual processing & Avoid eye contact & 35 & 11 \\
\cline { 2 - 4 } & Avoid reading and writing & 49 & 22 \\
\hline Auditory processing & Responds to name & 38 & 26 \\
\cline { 2 - 4 } & Love noises & 34 & \\
\cline { 2 - 4 } & Irritate by sounds & & \\
\hline
\end{tabular}

Above table show the frequency of sensory-motor problems in children with autism spectrum disorder. In data analysis of vestibular problems 7 children disliked play, 13 were clumsy and 7 children did not like to jump on swings and 38 children spins constantly. Analysis of proprioceptive problems showed that 24 children had stiff and uncoordinated movements, only 9 had difficulty in getting out of chair and 19 were unable to grasp pencil. A tactile problem showed that 28 children disliked textures, 28 disliked brushing, combing, 30 showed no reaction to stimuli and 24 craves touch. Visual processing disorder showed that most of the children, 41 avoid eye contact, and 35 children avoid reading and writing. Auditory processing disorder analysis showed that 11 children did not respond to name, 38 children loved noises, most of the children (34) irritate by sounds.

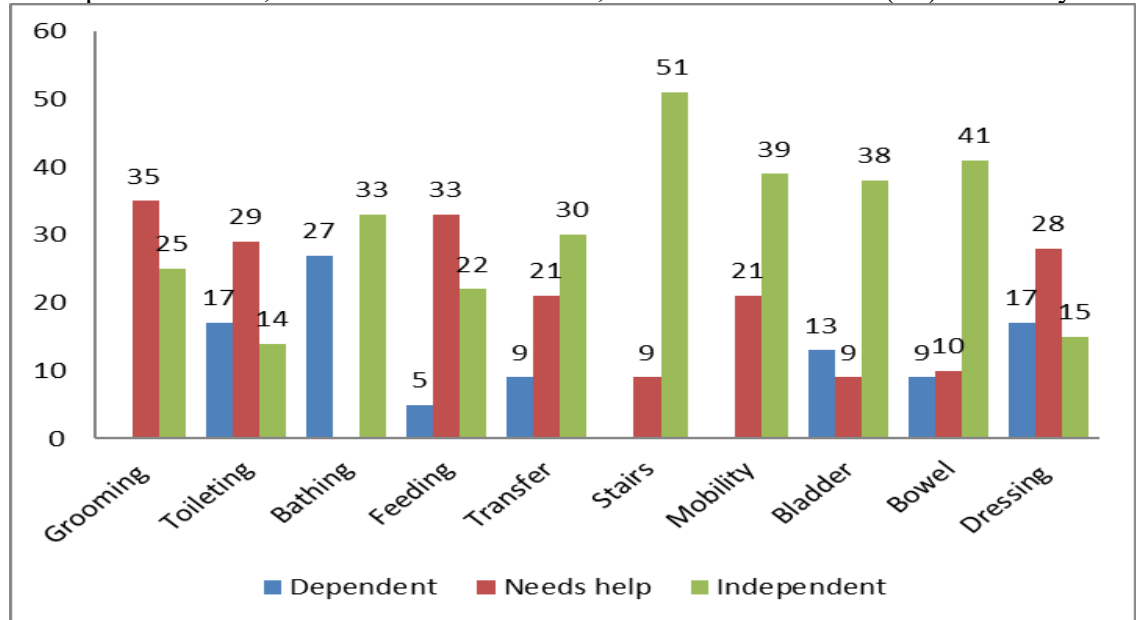

Figure3: Bar chart of frequency of Activities of Daily Living children with ASD

Statistical Analysis of Activities of Daily Living showed that in case of analysis of Bowels/Bladder 9 children had Bowel incontinence, 10 occasional accidents and maximum 41 children had Bowel continence, 
most of the children 38 had bladder continence, 9 children had occasional accident and 13 were incontinent. Analysis of Grooming showed that in case of dressing 17 were dependent, 28 children needed help in dressing and 15 were independent in dressing. In case of grooming, most of the children 35 needed help in grooming and 25 were independent. Analysis of Bathing showed that in Toilet use 17 were dependent, 29 needed help and 14 were independent, In case of Bathing 27 were dependent, 33 were independent. Feeding analysis showed that minimum children 5 were unable; mostly children 33 needed help and 22 were independent. In Mobility, 21 children needed minor help in transferring; most of the children 39 were independent in the transfer. In using stairs 5 needed help and a maximum number of children 51 were independent in using stairs.

Table 5.3: Correlation between Barthel Index scale and sensory-motor issues.

\begin{tabular}{|l|l|l|l|l|}
\hline & Mean & St. deviation & r & P \\
\hline Total score of vestibular problem & 6.1333 & 0.74712 & 0.23 & -157 \\
\hline Total score of proprioceptive problems & 4.7667 & 0.72174 & 0.656 & -0.059 \\
\hline Total score of tactile problems & 6.1667 & 1.02786 & 0.001 & $.404^{* *}$ \\
\hline Total score of visual processing & 2.7333 & 0.75614 & 0.671 & 0.056 \\
\hline Total score of auditory problems & 5.6833 & 0.98276 & 0.009 & $-.332^{* *}$ \\
\hline
\end{tabular}

ADLS (Mean= 13.966667, St. Deviation= 2.99132)

The table showed that the tactile system is highly negatively significantly $\left(.404^{* *}\right)$ correlated with ADLs $(\mathrm{r}=-0.001)$. Auditory processing is negatively significant $\left(-.332^{* *}\right)$ in correlation with ADLs $(\mathrm{r}=-0.009)$.

\section{Discussion}

The aim of the study was to assess how daily living Tasks are affect by sensory-motor problems in children with autism spectrum disorder (ASD). Children with ASD have sensory - motor problems which affect the performance of daily living tasks like grooming, bathing, toileting, hygiene, feeding dressing, etc. In the domain of self-care of this vulnerable population, its sensory-motor aspect remains to be characterized. The total sample consisted of 60 children with autism spectrum disorder in which there were 40 male and 20 female. The children were in the age range of 5-12. The present study explains poorest expected ADLS of ASD's group. The result of the research shows the calculated correlations between sensory-motor performance and daily living tasks. These results illustrated that vestibular system, Proprioceptive system, visual processing system were not correlated significantly with ADLS. While tactile processing and auditory system processing was significantly correlated with ADLS. According to these correlations children having difficulties in the tactile processing, integration found it difficult to perform the tasks which involve fine motor skills for example toileting, grooming, bathing, and dressing. (Carolanne Mattard-Labrecque,et al., 2013) This pilot study aimed to compare sensory processing, motor skills and adaptive behaviors in children with a double diagnosis of Autism Spectrum Disorder (ASD) and Attention Deficit Hyperactivity Disorder (ADHD) (ASD+ADHD) with children with ADHD alone and to examine the association of sensory processing and motor skills with adaptive behaviors (self-care) Thirty kids aged 5-14 years determined to have ASD and ADHD $(n=13)$ or ADHD $(n=17)$ were assessed on their sensory processing and motor skills and adaptive behaviors. Investigation of covariance looked at the gatherings on these measurements. Connection examinations analyzed the relationship between sensory processing and motor skills and adaptive behaviors. Contrasted with children with ADHD alone, kids with ASD and ADHD had less fortunate aptitudes in sensory preparing $(p<0.001)$, motor $(p=0.001)$ and adaptive behaviors $(p<0.001)$. For all children, expanded self-rule in self-care was connected with better sensory processing $(\mathrm{p}<0.001)$ and motor skills $(\mathrm{p}=0.002)$. Children with ASD+ADHD have poorer sensory processing, motor and adaptive behavior than those with ADHD alone. Sensory processing and motor problems were adversely connected with independence in self-care. Intercessions intending to improve sensory processing and motor skills and self-governance in selfcare ought to wind up significant focuses for these children ${ }^{24}$. (Vanessa Hus Bal et al,. 2015) this study was conducted on Daily living skills in individuals with autism spectrum disorder from 2 to 21 years of age this examination was directed on Daily living abilities in people with autism spectrum disorder from 2 to 21 years of age. Daily living skills (DLS) for example, personal hygiene, meal preparation, and money management, are important to independent living. Research recommends that numerous people with autism spectrum disorder display disabilities in daily living activities in respect to their psychological abilities. This examination inspected indicators of daily living skills achievement and directions of daily living skills in a longitudinal example alluded for conceivable autism spectrum disorder and pursued from 2 to 21 years of age. Reliable with past examinations, members with autism spectrum disorder and non-spectrum diagnosis indicated nonstop development of daily living skills all through childhood and pre-adulthood. Early youth nonverbal mental age was the most grounded indicator of daily living activities fulfillment for both diagnostic groups. There are two particular directions of daily living skills development for children with autism spectrum disorder. Expertise levels for the two groups of young adults with autism spectrum disorder remained significantly beneath age level desires. While the "High-DLS" bunch picked up roughly 12 years in daily living skills from T2 to T21, the "Low-DLS" group's daily living skills improved 3-4 years over the 16-to 19-year study period. Nonverbal 
mental age, receptive language, and social-communication hindrance at 2 years anticipated High-versus LowDLS bunch participation. Accepting more prominent than $20 \mathrm{~h}$ of parent-executed mediation before age 3 was likewise connected with daily living skills trajectory. Results recommend that daily living skills ought to be a focal point of treatment plans for people with autism spectrum disorder, especially adolescents changing to young adult $\mathrm{t}^{25}$.

Associations have been found among unusual sensory responding and performance of daily living skills such as grooming, bathing, and bedtime behaviors. Sensory processing patterns are also associated with sleep difficulties, eating and mealtime behavior, play and motor skills (Ayres, 1985) ${ }^{26}$. Linda Mechling, Frank J. Sansosti (2013) studied on awareness on how the latest technologies can assist the individuals to perform their daily living tasks. Individuals with autism spectrum disorder have sensory motor problems which make it difficult for them to perform their daily tasks and because of this most of the individuals with ASD cannot perform their daily task and rely on their care giver or assistant. According to the study technology can be used to make it easier for the individuals with autism to perform their task independently ${ }^{27}$. Our result was also in agreement with the study conducted by Gisel, E. (2010) studied how sensory motor problems affect mealtime in children with autism spectrum disorder in which they found that children with autism have more mealtime problem than their siblings because of their sensory issues even though they had limited food variety. Children with ASD had more eating problems as an infants. Older children tended to have fewer problems than younger children. ${ }^{28}$ Madhukar H. Trivedi, Carolyn R. Garver, Bruce D. Grannemann 2006 studied 4 different modalities of tactile, auditory, visual and oral to check out the sensitivity response. All the 4 modalities were examined separately at both level of sensory seeking and avoidance. Individuals with autism showed change in response of sensory stimulus. The change in sensory avoidance was not remarkable but the change in response to sensory seeking in auditory and visual stimuli ${ }^{29}$.

\section{Conclusion}

It is concluded that children with autism spectrum disorder (ASD) shows poor sensory and motor response and they were having difficulties in performing their daily living. Most of the individuals were having difficulties because of sensory motor problems in tactile and auditory processing and thus causes poor performance and dependence in daily Living task. Problems in tactile processing indicate children may have difficulties in tasks such as dressing, grooming, bathing toileting, etc.

\section{References}

1. American Psychiatric Association. (2013). Copyright: Diagnostic and statistical manual of mental disorders (DSM-V-TR) (5th ed.). Arlington, VA: American Psychiatric Publishing

2. Tom T. Shimabukuro Scott D. Grosse Catherine Rice. Medical Expenditures for Children with an Autism Spectrum Disorder in a Privately Insured Population. Journal of autism and developmental disorders. March 2008, volume 38, issue 3 .

3. (Baio J. Prevalence of autism spectrum disorder among children aged 8 years-autism and developmental disabilities monitoring network, 11 sites, United States, 2010.)

4. Roseann C. Schaaf Teal Benevides. An Intervention for Sensory Difficulties in Children with Autism: A Randomized Trial. 2013 Nov 10, 2014; 44(7): 1493-1506. doi: 10.1007/s10803-013-1983-8

5. Bailey A, Phillips W, Rutter M. Autism: towards an integration of clinical, genetic, neuropsychological, and neurobiological perspectives. J Child Psychol Psychiatry. 1996;37:89 -126

6. Bailey A, Le Courteur A, Gottesman I. Autism as a strongly genetic disorder: evidence from a British twin study. Psychol Med. 1995;25:63-77

7. Lopez-Rangel E, Lewis ME. Loud and clear evidence for gene silencing by epigenetic mechanisms in autism spectrum and related neurodevelopmental disorders. Clin Genet. 2006;69: 21-22

8. Veenstra-Vanderweele J, Christian SL, Cook EH Jr. Autism as a paradigmatic complex genetic disorder. Annu Rev Genomics Hum Genet. 2004;5:379 - 405

9. Grace T. Baranek, Linda R. Watson. hyporesponsiveness to Social and Nonsocial Sensory Stimuli in Children with Autism, Children with Developmental Delays, and Typically Developing Children. Dev Psychopathol. 2013 May; 25(2): 307-320. doi: 10.1017/S0954579412001071Susan Dodd. Understanding Autism. Introduction

10. Penelope Hannant, Teresa Tavassoli, Sarah Cassidy. The role of sensorimotor difficulties in autism spectrum condition. Published on Aug 10, 2016. doi: 10.3389/fneur.2016.00124

11. Thelen E, Smith LB. Dynamic systems theories. Handbook of child psychology. 1998.

12. Lewkowicz DJ, Ghazanfar AA. The emergence of multisensory systems through perceptual narrowing. Trends in cognitive sciences. 2009 Nov 1;13(11):470-8.

13. Dunn W, Myles BS, Orr S. Sensory processing issues associated with Asperger syndrome: A preliminary investigation. The American Journal of Occupational Therapy. 2002 Jan 1;56(1):97-102. 
14. Piek JP, Dyck MJ. Sensory-motor deficits in children with developmental coordination disorder, attention deficit hyperactivity disorder and autistic disorder. Human movement science. 2004 Oct 1;23(3-4):475-88.)

15. Sensorimotor skills.North Shore Pediatric Therapy

16. Penelope Hannant, Teresa Tavassoli, Sarah Cassidy. The role of sensorimotor difficulties in autism spectrum condition. Published on Aug 10, 2016. doi: 10.3389/fneur.2016.00124

17. Emmanuelle Jasmin, Melanie Couture, Patricia Anne Mckinley, Greg Reid (2009). Sensori-motor and Daily Living Skills of Preschool Children with Autism Spectrum Disorders. Journal of Autism and Developmental Disorders 39(2):231-41.

18. Occopational Therapy Practice Framework:Domain and Process. American Journal of Occupational Therapy.2002;56:609-39.

19. Interactive Autism Network.2007.

20. Ricon, Tsameret, Rachel Sorek, and Batya Engel Yeger. "Association between sensory processing by children with high functioning Autism Spectrum Disorder and their daily routines." The Open Journal of Occupational Therapy 5.4 (2017): 3.

21. Zobel-Lachiusa J, Andrianopoulos MV, Mailloux Z, Cermak SA. Sensory differences and mealtime behavior in children with autism. American Journal of Occupational Therapy. 2015 Sep 1;69(5):6905185050p1-8.

22. Tomchek SD, Little LM, Dunn W. Sensory pattern contributions to developmental performance in children with autism spectrum disorder. American Journal of Occupational Therapy. 2015 Sep 1;69(5):6905185040p1-0.

23. Mattard-Labrecque C, Amor LB, Couture MM. Children with autism and attention difficulties: A pilot study of the association between sensory, motor, and adaptive behaviors. Journal of the Canadian Academy of Child and Adolescent Psychiatry. 2013 May;22(2):139.

24. Bal VH, Kim SH, Cheong D, Lord C. Daily living skills in individuals with autism spectrum disorder from 2 to 21 years of age. Autism. 2015 Oct;19(7):774-84.

25. Ayres KM, Mechling L, Sansosti FJ. The use of mobile technologies to assist with life skills/independence of students with moderate/severe intellectual disability and/or autism spectrum disorders: Considerations for the future of school psychology. Psychology in the Schools. 2013 Mar; 50(3):259-71.

26. Renee Watling; Sarah Hauer. Effectiveness of Ayres Sensory Integration ${ }^{\circledR}$ and Sensory-Based Interventions for People with Autism Spectrum Disorder: A Systematic Review. American Journal of Occupational Therapy, September 2015, Vol. 69, 6905180030. https://doi.org/10.5014/ajot.2015.018051

27. Kevin Michael Ayres, Linda Mechling, Frank J. Sansosti. THE USE OF MOBILE TECHNOLOGIES TO ASSIST WITH LIFE SKILLS/INDEPENDENCE OF STUDENTS WITH MODERATE/SEVERE INTELLECTUAL DISABILITY AND/OR AUTISM SPECTRUM DISORDERS: CONSIDERATIONS FOR THE FUTURE OF SCHOOL PSYCHOLOGY, 24 January 2013, https://doi.org/10.1002/pits.2167

28. Geneviève Nadon, Debbie Ehrmann Feldman, Winnie Dunn, Erika G Gisel. Mealtime problems in children with Autism Spectrum Disorder and their typically developing siblings: A comparison study. January 2011,Autism 15(1):98-113,DOI:10.1177/1362361309348943

29. Kern JK, Trivedi MH, Garver CR, Grannemann BD, Andrews AA, Savla JS, Johnson DG, Mehta JA, Schroeder JL. The pattern of sensory processing abnormalities in autism. Autism. 2006 Sep; 10(5):480-94. 